\title{
BMJ Open Fit for purpose? OrganisationaL prOdUctivity and woRkforce wellbeIng in workSpaces in Hospital (FLOURISH): a multimethod qualitative study protocol
}

\author{
Frances Rapport, ${ }^{1}$ Emilie Auton, ${ }^{\oplus 1}$ John Cartmill, ${ }^{2}$ Jeffrey Braithwaite, ${ }^{\circledR} 1$ \\ Patti Shih, ${ }^{\oplus 1}$ Anne Hogden, ${ }^{\oplus 1}$ Robyn Clay-Williams ${ }^{\oplus 1}$
}

To cite: Rapport F, Auton E, Cartmill J, et al. Fit for purpose? OrganisationaL prOdUctivity and woRkforce wellbelng in workSpaces in Hospital (FLOURISH): a multimethod qualitative study protocol. BMJ Open 2019;9:e027636. doi:10.1136/ bmjopen-2018-027636

\section{- Prepublication history for} this paper is available online. To view these files, please visit the journal online (http://dx.doi. org/10.1136/bmjopen-2018027636).

Received 2 November 2018 Revised 4 February 2019 Accepted 13 March 2019
Check for updates

(C) Author(s) (or their employer(s)) 2019. Re-use permitted under CC BY-NC. No commercial re-use. See rights and permissions. Published by BMJ.

${ }^{1}$ Australian Institute of Health Innovation, Macquarie University, Sydney, New South Wales, Australia

${ }^{2}$ Macquarie University Hospital, Macquarie Park, New South Wales, Australia

Correspondence to

Dr Emilie Auton;

emilie.auton@mq.edu.au

\section{ABSTRACT}

Introduction Workspaces are socially constructed environments where social relationships are produced, reproduced, challenged and transformed. Their primary function is to support high-quality service delivery to the benefit of 'patients'. They are also settings where employees can work effectively, in a safe and healthy environment, delivering a high-quality service according to a 'Work-As-Done' rather than a 'Work-As-Imagined' model. However, hospital design is currently based on a managerial understanding of work accomplishments, often falling short of understanding what is actually happening on the ground. Furthermore, the research landscape lacks rigorous assessment of these complex sociological and health research concepts, either within the Australian context where this protocol is set, or internationally. This paper describes an innovative protocol aimed at examining healthcare employees' and organisations' concerns and beliefs in workspace design. It outlines research investigating the effect of workspace use on productivity, health and safety and worker satisfaction, to clarify Work-As-Done, while creating healthy and more fulfilling environments.

Methods This is a proof-of-concept study, taking place between June 2018 and April 2019, employing a multimethod, qualitative approach for in-depth assessment of one Australian, private, university hospital environment, using as its 'case' the Gastroenterology Surgical Unit. It involves (1) observations and informal interviews (shadowing) with employees and patients as they traverse hospital spaces and (2) visual data of spatial use. Fieldnotes will be analysed thematically, and visual data analysed using a predefined schematic framework (a visual taxonomy). Overarching themes and categories will be considered corroboratively, mixing visual and textual data to build an iterative and dynamic picture.

Ethics and dissemination Ethical considerations will be discussed, while approval has been granted by the University's Human Research Ethics Committee (HREC/5201800282), along with Governance approved by the Health Clinical Research Executive (CRG2018005). Study results will be disseminated through publications, research conferences and public reports.
Strengths and limitations of this study

- First Australian case-study of a surgical unit examined in terms of its workspaces and practices.

- First study to investigate the use of mobile methods to understand Work-As-Done by healthcare professionals when delivering patient care with the potential for a new method for use in resilient healthcare research.

Detailed understanding of the strengths and challenges of Work-As-Done with implications for improving hospital environments for the betterment of the case site's healthcare workforce and patients.

- As a proof-of-concept study, the small sample size (small, fixed team) and single site (private, affluent, metropolitan hospital serving a homogenous population group) limit the scope of the findings. Even so, the study is designed to generate in-depth, nuanced data with aspects that are transferrable to other settings, while bringing hospital management and staff into a stronger research collaboration.

\section{INTRODUCTION}

Workspace design

Workspaces are socially constructed environments and active parts of our lives. ${ }^{1}$ They are places where 'social relationships are produced, reproduced, contested and transformed' ( $p$ 532). ${ }^{2}$ The primary function of workspaces is to support a workforce in delivering a service or product of the highest quality $^{34}$ to the benefit of a client group (also known as the 'customer', 'user' or, in the case of a healthcare workspace, the 'patient'). They are also places where employees should feel safe and healthy. ${ }^{34}$ When these primary factors align, secondary benefits are psychosocial well-being,${ }^{5-7}$ productivity $^{8}$ and economic viability, ${ }^{9-11}$ and when managed effectively, workspaces can be said to be better able to cope with the challenges that characterise 
the work being undertaken within them, often described as 'resilient' space. ${ }^{3}$ Studies of workspace design, across different industries, have predominantly concentrated on ergonomics, ${ }^{12} 13$ including stress and safety in the workplace. ${ }^{14}$ They have assessed the effect of stress on individual and organisational productivity and job satisfaction, ${ }^{15}$ leading to new sociobehavioural interventions. ${ }^{16}$ The Australian National Workplace Health Project, ${ }^{16}$ for example, concentrated on introducing new behavioural interventions such as healthy eating, to enhance fitness and well-being, while work in stress and safety has led to new definitions of psychosocial factors that affect strain and employee health. ${ }^{14}$ Wilson et $a l^{17}$ and Allen et $a l^{18}$ examined absenteeism and job satisfaction with strong correlations found between higher absenteeism and workplace stress, and between lower job satisfaction and lower organisational commitment and turnover. ${ }^{18}$

\section{Reduced psychosocial well-being}

Reduced psychosocial well-being has been described as an antecedent to lower productivity and customer satisfaction, ${ }^{15} 19$ and is also linked to psychological withdrawal. ${ }^{15}$ Having examined psychosocial well-being in some detail, Vischer ${ }^{14}$ urges us to turn our attention to the physical environment, which is underexamined-its layout, makeup and objects within it that are affected by its design, and these act as strong indicators of a workforce's productivity, health and safety; what Vischer calls 'environmental psychology' ( $p$ 175). ${ }^{14}$ This view is strengthened by the work of Veitch $e t a l^{20}$ who noted that most research in the physical environment fails to lead to clearer workspace design-leaving this still a largely uncharted territory. ${ }^{16}$

\section{Increases in working hours}

In 2015, in Australian healthcare where this study is situated, over 460000 registered healthcare professionals are actually working a 42-72 hour-week, rather than a 38 hour-week (the prescribed number of working hours for optimal health and safety), with one in two doctors described by the Australian Medical Association as working unsafe hours. ${ }^{21} 22$ With a growing number of hours in work, good workspace design is crucial to people's safety, productivity and job satisfaction. ${ }^{23}$ According to the Productivity Commission's Report on Government health services, ${ }^{24}$ at least 100 medical disasters occur each year causing death or serious harm. This includes unsafe operations, removal of wrong body parts and foreign bodies remaining after operations. Overstretched staff, crowded hospitals and lengthy waiting times, as well as a lack of individualised, appropriate care exacerbate the prevalence of such errors, with 82 sentinel events recorded in NSW alone between 2015 and 2016. Yet, despite these statistics, healthcare employees who are vital players in keeping the nation safe, are not fully engaged in hospital planning ${ }^{25}$ and do not report on their workspace needs or the needs of their patients.

\section{Increases in medical errors and error reporting}

Longer work hours lead to more medical errors with 1-in-10 patient encounters currently associated with a medical error (unsafe procedure or wrong drug). ${ }^{22} \mathrm{Up}$ to 18000 Australians die unnecessarily each year from errors, ${ }^{26}$ also estimated as the third leading cause of death in the USA. ${ }^{27}$ Errors result from tiredness, inefficiencies in the workplace and ill-placed technology and objects, leading the workforce to find themselves under increasing stress. ${ }^{222}{ }^{28}$ Error reporting is also on the increase globally, which in turn leads to a whistleblowing society that is said to demotivate the workforce. ${ }^{27}$ A demotivated workforce can make further errors (such as an incorrect operation). ${ }^{29}$ In Australia, a sudden rise in doctors' mental health problems, including burnout and suicide, has been characterised as an 'epidemic' and 'crisis ${ }^{30}$ pronounced by the ageing Australian population, with hospitalisation rates growing yearly, especially for the over $65 \mathrm{~s},{ }^{31}$ and hospitals stretched by limited resources. ${ }^{32}$

We need to urgently address the challenges outlined here, namely reduced psychosocial well-being among the workforce (including increased levels of stress, inefficiency and tiredness), and increased working hours and as a result more medical errors and error reporting. The proposed study offers a unique opportunity to directly address these significant challenges, through the translation of findings from an innovative investigation of workspace functionality and the effect of workspaces on workforces, into practical outputs to improve and ensure workspaces are safer, healthier and more productive environments for people to work together in, which in turn will reduce errors, improve psychosocial well-being and professionals' sense of productivity and effective practice.

The hospital operating theatre, where this study will be situated, has been chosen as a site for in-depth and observational investigation, as a result of it being a space where highly technical work takes place, and where collaborative and harmonious team work is crucial to staff and patient safety, well-being and productivity. Operating theatres, where the use of space is highly regulated, are also one of the most inaccessible spaces in the hospital, making them a challenging test-bed for spatial investigation. To elaborate, Fox $^{33}$ proposed that there are three 'circuits of hygiene' in operating theatres that serve to protect the surgical space and those working within it-the instrument circuit, the staff circuit and the patient circuit. These all help to maintain the sterile environment, so that surgery may be performed safely. The instrument circuit ensures that the route of clean and sterile equipment remains separate from contaminated equipment. The staff circuit refers to the staff-only access points that regulate entry and exit to theatres. The patient circuit is the limited access to patient holding-rooms and the anaesthetic room. Fox argued that the architecture acts as a reminder to staff to follow sterile procedures; 'it can be "read" and contributes to the routines necessary for safe, sterile surgery' ( $p$ 656) ${ }^{33}$ While there is a growing body of knowledge about social relationships in operating 
theatres, ${ }^{33-35}$ we will use mobile methods to explore a broader suite of issues, namely workforce well-being and productivity as well as patient and professional safety.

Good workspace design is key to the quality of people's work, their safety, and well-being (happiness, health and sense of productivity),${ }^{23}$ and yet the healthcare workforce, a sector where effective function is crucial to national wellbeing, is rarely consulted about how workspaces should function effectively. Consequently, we lack vital knowledge about optimal hospital design in line with healthcare workforce needs, to impact positively on employees, and ultimately, high-quality, safe, care delivery.

\section{Conceptual framework}

In complex adaptive systems such as healthcare ${ }^{36}$ WorkAs-Done (WAD) on the front line of patient care is always different from Work-As-Imagined (WAI) by those who design workspaces. WAI and WAD are concepts derived from the field of resilient healthcare. ${ }^{37-39}$ Assumptions about WAD from those doing WAI are often misguided, because they are founded on a misunderstanding of the healthcare environment. WAI is how the work is understood by those who are separated from the work by time or space (eg, policymakers, legislators and governments). WAI is typically a simplified view of the work that goes on, in this case in a hospital setting, but does not account for the myriad small and large adjustments that healthcare professionals must make in their everyday practice to get the work done (WAD). Due to misalignment between WAI and WAD, when rules are mandated or prescribed in terms of WAI, they can be difficult (or even impossible) to follow by clinicians working at the coalface. WAD, within a hospital setting, defines people's perceptions of their roles, responsibilities and activities, including the small and large adjustments that people make to their daily work in order to function effectively, despite the fragmented, complex, resource-restrained and challenging circumstances in which they often find themselves. ${ }^{4041}$ WAD includes not only work practices and perceptions, but also systems and settings, individual and group work patterns, approaches to policy and practice, 'workarounds' and accommodation of daily exigencies and aspirations for improvement. WAD in hospitals is frequently epitomised by a workforce's struggle to 'make do' in unsuitable spaces, or to find ways to circumvent, deviate from, or overcome challenges that arise. This can lead to unsafe practices and demoralisation, and until hospital spaces are adequately matched to the needs of the workforce, this could continue to lead to expensive, impactful mistakes and a lowering of standards of care. ${ }^{36}$

Studies on workspaces in Australia and internationally, have overwhelmingly focused on managers' or customers' perspectives, while the views and experiences of employees is underacknowledged.$^{42}$ Rarely does research encourage employees to answer questions about their work environments, and little has been done worldwide to map healthcare landscapes in terms of productivity, safety and personal health and well-being. ${ }^{2} 614$
Furthermore, research on a human scale often prioritises questionnaires and focus group data, collected outside the workplace, rather than understanding work in situ. Without immersion in the landscape-tightly and loosely coupled ecosystems-we cannot fully comprehend how people function or understand WAD.

\section{Mobile methods}

Mobile methods is an innovative methodology that involves researchers undertaking data collection through observations 'on the hoof' alongside research participants. ${ }^{43}$ They are useful for capturing the increasing mobilities of people, goods and objects, and can offer innovative insights into people's sense of identity, interaction and power relationships. ${ }^{44}$ Mobile methods are also used to describe organisational structures, adaptation and complexity. In this study, we focus on a narrow aspect of mobile methods called 'shadowing', where a researcher 'follows selected people in their everyday occupations for a time' (p17),${ }^{45}$ and in the process, where a researcher asks people questions, often on the move, and while listening and observing what they do and to whom they speak. Shadowing allows researchers to describe and understand patterns of social, occupational and spatial engagement. Shadowing can be accomplished using a variety of forms of travel (eg, driving or taking the train), but in this study we have selected walking, for its slow, variable and flexible nature, and as a result of its appropriateness to a hospital context. Shadowing will allow us to understand the complexities of the surgical work environment, how it varies, how work is connected within and beyond the operating theatres, and how work is achieved.

There are several advantages of shadowing. First, shadowing allows a researcher to observe participants' spatial practices in situ while simultaneously accessing their experiences and interpretations. ${ }^{46}$ This means a researcher can assess WAD in clinical workspaces because they can witness what people actually do in their everyday working life rather than what people say they do in experience-distant situations (such as captured in surveys or interviews). Second, mobility is such a pervasive feature of our lives, and is constitutive of modern medical work, where, for instance, a consultant surgeon may inhabit and transverse multiple spaces in order to conduct her work; a kind of choreographed set of surgical and ancillary practices. Third, because researchers engage participants in situ and encourage reflection, mobile methods have the potential to initiate or advance professional growth and social change. ${ }^{45}$ Finally, shadowing is an educational technique as well as a research method, particularly in the medical and nursing fields. In a surgical context, for instance, a nurse trainee may be assigned to an experienced scout nurse whom she follows for a period of time in order to observe what the role entails. In this context, shadowing is the method used to ensure WAD is produced and reproduced between experienced healthcare professionals and newcomers. This means that the researcher holds a dual role, that of a research participant observing 
others' work, and that of an observer walking in the footsteps of a medical trainee who is learning about WAD. As both researcher and newcomer learn about WAD, through the process of shadowing, the method has the potential to generate deeper and more nuanced insights into the context of workspace use and professional practice, in comparison to other qualitative methods.

This study protocol outlines the methodological approach of a proof-of-concept study of WAD in a single private Australian teaching hospital's Gastroenterological Surgical Unit (from now on known as 'the case'). We will also outline some of the ethical issues specific to mobile methods and the ways in which we will manage these challenges. Using an innovative research design and a unique site, the proposed study directly addresses key gaps in current research evidence, while examining spatial design of workspace and practice. A dedicated study researcher will work alongside case members to ensure a thorough assessment of how people move through, use and adapt to different spaces, and the role space plays in people's sense of productivity, good health and well-being and safety.

\section{METHODS AND ANALYSIS \\ Objective}

This research project will examine how spatial design of workspace hubs can influence workers' productivity, health and safety in one Australian hospital setting.

\section{Aims}

1. To clarify how the case workforce arrange, traverse and share workspace.

2. To reveal how different case members manage space and the objects within it.

3. To disclose individual and team views on WAI/WAD, productivity, health and safety and well-being.

4. To assess how enabling and disabling qualities of space affect WAD.

5. To define the complexities of this case, in terms of the impact of workspace on people's lives.

\section{Study design}

This is a multimethod, qualitative study with (1) qualitative observations of spatial use, (2) informal conversations using 'mobile methods ${ }^{43}$ with the case workforce and (3) the collection of images (photographs and architectural plans) of spatial layout of one hospital's gastroenterology surgical spaces, and associated work environments. This study uses mobile methods and visual data in order to most appropriately describe and image spatial functionality. The study will be conducted between June 2018 and April 2019.

\section{Sample and setting}

The case site is a private teaching hospital located within the bounds of an Australian university campus, co-located with a university medical faculty, comprising 182 beds, 12 operating theatres, two cardiac and angiogram suites as well as other facilities and amenities (eg, pharmacy, cafeterias and other leisure spaces and medical imaging suite). The hospital is a privately funded, affluent, metropolitan organisation that is dedicated to a culture of continuous improvement through research and education, making it an ideal pilot site for this study. This study will examine one small, fixed-member team, which offers a typical example of a team working in this setting, caring for a culturally homogenous, predominately English-speaking population. The composition of the gastroenterology team (including one to two consultant surgeons, two scout nurses, one scrub nurse, one anaesthesiologist and one anaesthetic nurse) is typical of these types of hospital arrangements. The surgical team typically comprised one colorectal surgeon for relatively straight-forward procedures (colonoscopies or hernia repairs); however, for more complex cases (eg, low anterior resection for rectal cancer), the team may include two colorectal surgeons who work together. The case site is a relatively modern hospital (completed 2010), with cutting-edge diagnostic and treatment technology such as fully integrated digital operating suites and electronic medical records. As a result, it is a good example of contemporary Australian hospital architecture. This proof-of-concept study will generate in-depth knowledge of one case, but by doing so, will not only create research strategies transferrable to other settings, but also bring the hospital and the university staff into a stronger research collaboration with the potential of expanding to other units, settings and wards, through shared knowledge and shared goals.

\section{Recruitment procedure}

\section{Group 1: Healthcare professionals}

Healthcare professionals will be recruited using purposive sampling. Prior to the start of shadowing, the research team will give a full briefing of the aims, methods and intended outcomes of the study to the core gastroenterology team, while other gastroenterology staff members will be invited to attend the meeting if they wish, so that they can raise any queries. At the end of the briefing, those willing to be involved will sign a consent form. We aim to recruit a range of healthcare professionals to ensure a wide variety of observations of spatial use and relationships.

Following any incidental interaction with members of the wider gastroenterology surgical team who were either not present at the meeting or were present but unwilling to consent at that time, the hospital staff member will be verbally informed about the study when they come into contact with the study researcher. If they elect to participate, they will sign a participant consent form retrospectively. If they elect not to participate they will not be observed as part of the study.

\section{Group 2: Patients}

Patients are not the primary focus of this study; nevertheless, in the course of shadowing fixed-team members, 
patients will be incidentally observed and as a consequence we will ensure recruitment is responsibly undertaken. Patients will be recruited using time-frame sampling. During the recruitment period, the first 20 patients who are due to undergo surgery with the gastroenterology team, aged $\geq 18$ years and able to speak English, will be contacted in the order in which they are put onto the surgical team's surgery list. Time-frame sampling removes opportunistic recruitment of patients, and any researcher or hospital selection bias. It is a valuable technique when researchers are unable to predefine the sample of patients who will be attending the hospital clinic at any one time, setting an important framework around recruitment. A dedicated clinical liaison officer will undertake the recruitment and consent process on behalf of the study team in order to remove any researcher involvement until post consent. Recruitment and consent will take place prior to the scheduled day of a patient's surgery. On the day of surgery, the dedicated study researcher will introduce herself to the patient, verbally reconsent the patient and, if necessary, collect the signed study consent form.

This study will explore how staff members negotiate and move through space alongside the gastroenterology team members being shadowed. The data collection process will remain consistent with the stated aims and objectives. The researcher will not be collecting information of a personal, identifiable nature, nor any clinical details about patients and cases be recorded.

\section{Data collection}

\section{Shadowing and fieldnotes}

Mobile methods will provide insights into the experiences of the workforce, using the studied space, and how healthcare professionals perceive that the workspace enables or hampers their work. Understanding how staff members construct their workspace during daily routines, interactions and movement is a vital element of clarifying workspace use (aims 1-5). Mobile methods will support this assessment, enabling the researcher to move through space with others or alone, while watching and learning how surgeons, nurses, trainee doctors, students, anaesthetists, administrative staff and others from the gastroenterology team interact with one another, other hospital staff members and patients. Mobile methods will be undertaken by a dedicated study researcher (EA) for approximately 50 hours in total at different times of the day and on different days, to ensure naturalistic behaviour patterns are observed. It is important to note that the dedicated study researcher has no prior relationship with participants. This will enable a detailed examination of WAD, and the use and influence of space on routine, daily healthcare practices. This includes how staff converse with one another and with patients, and how staff interact in and across a variety of rooms and areas. Spaces may include surgical theatres, cafeterias and other leisure spaces, wards, corridors, waiting rooms and meeting rooms. Consistent with mobile ethnographic approaches ${ }^{4347}$ data quality will be strengthened through a rigorous recording process; brief notes will be handwritten while shadowing, then more detailed fieldnotes will be typed up within a few days of the shadowing. ${ }^{48}$ The data generated from shadowing will be spatially contextualised in pictorial or written forms in a notebook in order to take advantage of a mobile, place-sensitive method.

\section{Photographs, drawings and architectural plans}

The purpose of this component is to highlight the layout of workspace, variations between spaces (number, shape, location, size and open and closed areas), comparing the look of different spaces (eg, communal and private) and noting spatial use (aims 1, 2 and 4). The dedicated study researcher will create drawings and diagrams showing spatial layout and function, obtain the architectural plans of the areas that participants work in and take photographs of shared and private spaces, to clarify spatiality in hospital settings. This is in line with this team's prior work in the area, undertaken in primary and community care settings. ${ }^{27}$ Photographs and architectural plans will allow for a better understanding of how participants see, think, feel and memorise experience. ${ }^{27}$ Any images of individuals (healthcare professionals or patients) will be deidentified or destroyed.

\section{Data analysis}

Fieldnotes will be analysed using thematic analysis techniques $^{49}$ with two experienced qualitative analysts (primary and secondary) (EA and FR) working together to ensure the process is rigorous, discuss major and minor themes and their concomitant categories and arrive at consensus if variance occurs. Issues of significance will be categorised, with recurring categories organised into common themes. ${ }^{49}$ The secondary analyst will examine a subsample of data to ensure methodological veracity.

Photographic data and plans will be subjected to their own discrete analysis through visual techniques that are well published, and build on team members' previous research. ${ }^{2672650}$ The team will use a predefined schematic framework (a visual taxonomy), designed by the study lead and used across a variety of healthcare settings ${ }^{2} 67$ that takes account of visual content and context, but also of object clustering and positioning. The taxonomy examines the 'affect' of data (the feelings to which the photographs give rise), the frequency of spatial presentation, the way objects and spaces function and what photographs reveal about professionals' working patterns. The photographs will be considered for the different social and professional practices they display and how settings reflect group and individuals' workings.

Architectural plans and photographs will be analysed by team members through group-work activity. Applying the visual taxonomy, photographs will be compared and contrasted while analysis of architectural plans will include frequency distributions of the relationship between percentage space use and spatial arrangements, and assessments of objects in spaces (distribution, frequency and clustering). 
Analysis will encourage discussions of spatial arrangement and the use and usefulness of workspace. A theoretical case for optimal spatial arrangement, relating to the 'case', and based on the data collection stages, will be identified using Social Worlds Theory (SWT) ${ }^{51}{ }^{52}$ SWT will emphasise social aspects of space, including connectivity and belonging.

All data will be considered corroboratively to develop an in-depth understanding of the complete dataset, with data from the researcher's fieldnotes standing alongside the architectural plans and photographs. Team considerations of key themes and categories will be refined as data analysis continues.

\section{Patient and public involvement}

Patients and public representatives have influenced the design of this study through research that originated in the UK around the use of healthcare practitioner workspaces and their impact on professional and patient behaviour. ${ }^{267}$ In this study, patients will be the subject of surgical ward and surgery theatre use and, as a result, integral to data collection. Study findings will be reported and made available to participants in an executive summary document. This will contain relevant information about study processes, formatted for general consumption.

\section{ETHICS AND DISSEMINATION \\ Ethical considerations}

This study involves recruitment of the gastroenterology surgical team, gastroenterology surgical patients and any staff members from the case site who may interact with the aforementioned participant cohorts. Written informed consent will be acquired from all members of the gastroenterology team, staff members from the case site and patients. Given the potential vulnerability of patients in a surgical context, a dedicated clinical liaison officer will verbally consent patients about the study prior to the day of surgery and provide them with study information and a consent form. Patients will be required to return the signed consent form by post or electronically, or to bring it along to the ward. The clinical liaison officer will inform the dedicated study researcher of the details of the surgery so as to plan for observations. Before entering the surgical theatre, the dedicated study researcher will introduce herself to the patients, verbally re-consent patients and, if necessary, collect the signed consent form.

Ethics approval for an op-out approach was sought for this study due to the study team being unable to anticipate which hospital staff members would interact with the dedicated study researcher over any given observation period. In addition, the team developed the opt-out approach so that staff members who were incidentally observed would be made aware of the research taking place and would have the opportunity not to be involved. However, to mitigate against wherever possible, the research team will hold a staff meeting where they will outline the aims and practicalities of the study giving staff members the opportunity to ask questions or express concerns. In addition, the dedicated study researcher will wear a badge with her name and a 'research-inprogress' annotation. Posters will be placed around the surgical ward to provide details about the study as well as the study coordinator's contact details. Finally, following any incidental interaction with members of staff, the staff member will be verbally informed about the study, and given the opportunity to opt-out with data collected inadvertently, erased. The university's Human Research Ethics Committee responsible for assessing this study has granted approval for the study on this basis, with written consent gained retrospectively if necessary.

\section{Data storage and retention}

All study materials will be deidentified and data will be stored in password-protected computers belonging to the main university site or stored in a locked cabinet within secure offices. Only the named researchers on the team will have access to the data.

\section{Dissemination}

All data outputs will be disseminated widely, through academic and non-academic publications and presentations. Dissemination may include information about methodological developments, topic insights and gaps between WAD and WAI. Oral and poster presentations and a study report will be prepared for staff members at the site to enhance collaborative working practices and to ensure extended community engagement.

\section{RESEARCH SIGNIFICANCE, INNOVATION AND IMPACT}

This is the first Australian case-study of a Gastroenterological Surgical Unit examining workspace and practices, and the first study to apply this mix of methods within the Australian hospital's 'built environment'. This study, conducted in line with the study aims, is designed to lead to (1) stronger knowledge transfer between researchers, healthcare professionals and hospital managers (exchange and synthesis of ideas), (2) new understandings of spatial design and organisational productivity and (3) clearer recognition of the strengths and weaknesses of different spatial arrangements from the perspective of healthcare staff. The insights generated will demonstrate how to create a more productive hospital workforce, and safer and more fulfilling work environments for better patient care.

At the same time, the study will be rigorously designed to ensure long-term outcomes, based on well-validated research techniques, with national significance. Internationally, the field of resilient healthcare is in its infancy, in terms of methodological development, in particular in deriving suitable methods for understanding WAD by clinicians delivering patient care. The study is significant in investigating the use of mobile methods to understand WAD and has the potential for a new method in resilient healthcare research. By designing safe and harmonious 
work environments that support team communication and movement, we aim to build environments where surgery can function in an effective and safe manner.

Finally, this work will provide baseline data from which to develop future research in the field. A larger, multisite study would have the capacity to produce a set of clearly defined quality improvement measures (QIMs) for hospital design to drive positive change. QIMs will be a practical output for a larger, multisite study, which has the potential for national and international impact. By optimising workspace for more productive and empathic working practices, reducing healthcare workforces' stressors and enhancing staff members' physical and psychosocial well-being, the work promises to afford patients and healthcare professionals safer healthcare environments.

Contributors FR led the overall conceptualisation of the study design and contributed to the first and revised drafts of the manuscript. EA led the ethics approval procedure and co-led the first draft of the manuscript and revisions of the manuscript. JC contributed to the study design with primary clinical care expertise and to revising the manuscript. JB, PS and AH provided input to the study design, supported its conceptual framework and critically revised the manuscript for intellectual content. RC-W co-led the overall conceptualisation of the study design and contributed to the first draft and revisions of the manuscript. All authors provided final approval of the version submitted and accepted its accuracy and integrity.

Funding Funding was provided by a private donation through an Australian Gastroenterology Funding Stream and through an Australian Commission Funding Stream. JB's work is supported by multiple grants, including the National Health and Medical Research Council Partnership Grant for Health Systems Sustainability (ID: 9100002).

Competing interests $\mathrm{JC}$ is an employee of the case site and will not, therefore, be involved in data analysis.

\section{Patient consent for publication Not required.}

Ethics approval Ethical approval for the study has been granted by the main university's Human Research Ethics Committee (HREC/5201800282) and governance approval has been granted by the main university's Health and Clinical Research Executive (CRG2018005)

Provenance and peer review Not commissioned; externally peer reviewed.

Open access This is an open access article distributed in accordance with the Creative Commons Attribution Non Commercial (CC BY-NC 4.0) license, which permits others to distribute, remix, adapt, build upon this work non-commercially, and license their derivative works on different terms, provided the original work is properly cited, appropriate credit is given, any changes made indicated, and the use is non-commercial. See: http://creativecommons.org/licenses/by-nc/4.0/.

\section{REFERENCES}

1. Lefebvre H. The production of space. Oxford: Blackwell, 1991.

2. Rapport F, Doel MA, Elwyn G. Snapshots and snippets: general practitioners' reflections on professional space. Health Place 2007:13:532-44.

3. Hollnagel E, Braithwaite J, Wears RL. Resilient health care. Ashgate Publishing, Ltd: Surrey, 2013.

4. Wears RL, Hollnagel E, Braithwaite J. Resilient health care, Volume 2: the resilience of everyday clinical work. Surrey, UK: Ashgate Publishing, Ltd, 2015.

5. Cummins S, Curtis S, Diez-Roux AV, et al. Understanding and representing 'place' in health research: a relational approach. Soc Sci Med 2007;65:1825-38.

6. Rapport F, Doel MA, Greaves D, et al. From manila to monitor: biographies of general practitioner workspaces. Health 2006;10:233-51.

7. Rapport F, Doel MA, Jerzembek GS. "Convenient space" or "a tight squeeze": insider views on the community pharmacy. Health Place 2009;15:315-22.
8. OECD. Material resources, productivity and the environment: OECD Publishing, 2015.

9. Gesler W, Bell M, Curtis S, et al. Therapy by design: evaluating the UK hospital building program. Health Place 2004;10:117-28.

10. Kearns RA, Barnett JR. Consumerist ideology and the symbolic landscapes of private medicine. Health Place 1997;3:171-80.

11. Radley A, Taylor D. Images of recovery: a photo-elicitation study on the hospital ward. Qual Health Res 2003;13:77-99.

12. Berguer R. Surgery and ergonomics. Arch Surg 1999;134:1011-6.

13. Stone R, McCloy R. Ergonomics in medicine and surgery. BMJ 2004;328-1115-8.

14. Vischer JC. Towards an environmental psychology of workspace: how people are affected by environments for work. Archit Sci Rev 2008;51:97-108.

15. Harter JK, Schmidt FL, Keyes CL. Well-being in the workplace and its relationship to business outcomes: A review of the Gallup studies. In: Keyes C, Haidt J, eds. Flourishing: Positive Psychology and the Life Well-lived. Washington, USA: American Psychological Association, 2003.

16. Simpson JM, Oldenburg B, Owen N, et al. The Australian National Workplace Health Project: design and baseline findings. Prev Med 2000;31:249-60.

17. Wilson MG, Dejoy DM, Vandenberg RJ, et al. Work characteristics and employee health and well-being: Test of a model of healthy work organization. J Occup Organ Psychol 2004;77:565-88.

18. Allen DG, Weeks KP, Moffitt KR. Turnover intentions and voluntary turnover: the moderating roles of self-monitoring, locus of control, proactive personality, and risk aversion. J Appl Psychol 2005;90:980-90.

19. Schneider B, Hanges PJ, Smith DB, et al. Which comes first: employee attitudes or organizational financial and market performance? J Appl Psychol 2003;88(5):836-51.

20. Veitch JA, Charles KE, Farley KMJ, et al. A model of satisfaction with open-plan office conditions: COPE field findings. J Environ Psychol 2007;27:177-89.

21. Australian Bureau of Statistics. 2016 Census QuickStats. 2016 http:// quickstats.censusdata.abs.gov.au/census_services/getproduct/ census/2016/quickstat/036.

22. Australian Medical Association. Managing the Risks of Fatigue in the Workplace: 2016 AMA safe hours audit. 2017 https://ama.com.au/ article/2016-ama-safe-hours-audit.

23. Vischer J, Wifi M. The effect of workplace design on quality of life at work. Handbook of Environmental Psychology and Quality of Life Research. London: Springer, 2017.

24. Commision AGP. Report on Government Services 2018. Australia, 2018.

25. Gollop R, Whitby E, Buchanan D, et al. Influencing sceptical staff to become supporters of service improvement: a qualitative study of doctors' and managers' views. Qual Saf Health Care 2004;13:108-14.

26. Medical Error Australia. Medical Error Action Group, June Long Foundation. 2018 http://www.medicalerroraustralia.com/ (Accessed Nov 2018)

27. Makary MA, Daniel M. Medical error-the third leading cause of death in the US. BMJ 2016;353:i2139.

28. Kondro W. Medical errors increasing because of complexity of care and breakdown in doctor-patient relationship, physician consultant says. Canadian Medical Association Journal 2010;182:E645-6.

29. Braithwaite J, Hibbert PD, Jaffe A, et al. Quality of Health Care for Children in Australia, 2012-2013. JAMA 2018;319:1113-24.

30. Baigent M, Baigent R. Burnout in the medical profession: not a rite of passage. Med J Aust 2018;208:471-2.

31. McPake B, Mahal A. Addressing the needs of an aging population in the health system: the Australian Case. Health Systems \& Reform 2017;3:236-47.

32. Rollins A. Public hospitals stretched by rising demand. Australian Medicine 2014;26.

33. Fox NJ. Space, sterility and surgery: circuits of hygiene in the operating theatre. Soc Sci Med 1997;45(5):649-57.

34. Katz P. Ritual in the operating theatre. Ethnology 1981;20:335-40.

35. Tantchou J. Blurring Boundaries: Structural Constraints, Space, Tools, and Agency in an Operating Theater. Sci Technol Human Values 2013;39:336-73.

36. Braithwaite J, Clay-Williams R, Nugus $P$, et al. Health care as a complex adaptive system. In: Hollnagel E, Braithwaite J, Wears R, eds. Resilient health care. Surrey, UK: Ashgate Publishing Limited, 2013.

37. De Certeau M. The practice of everyday life. Berkeley: University of California Press, 1984.

38. Braithwaite J, Clay-Williams R, Hunt G, et al. Understanding resilient clinical practices in Emergency Department ecosystems. 
In: Braithwaite J, Wears R, Hollnagel E, eds. Reconciling work-asimagined and work-as-done. Surrey, UK: Ashgate Publishing Limited, 2016.

39. Clay-Williams R, Johnson J, Debono D, et al. The path from policy to practice: resilience of everyday work in acute settings. In: Waldorff S, Pedersen A, Ferlie E, eds. Palgrave Macmillan. London: Managing change - from health policy to practice, 2015.

40. Braithwaite J, Wears R, Hollnagel E. Resilient health care: reconciling work-as-imagined and work-as-done. Surrey, UK: Ashgate Publishing Limited, 2016.

41. Hollnagel E, Braithwaite J, Wears R. Resilient health care. Surrey, UK: Ashgate Publishing Limited, 2013.

42. Willis J, Goad P, Lyon C, et al. Designing for wellbeing: realizing benefits for patients through best practice hospital design. Melbourne: Australian Research Council, 2011.

43. Büscher M, Urry J, Witchger K. Mobile methods. New York, USA: Routledge, 2010.

44. Sheller M, Urry J. The new mobilities paradigm. Environment and Planning A: Economy and Space 2006;38:207-26.
45. Czarniawska B. Shadowing and other techniques of doing fieldwork in modern societies. Copenhagen, Denmark: Copenhagen Business School Press, 2007.

46. Kusenbach M. Street phenomenology: the go-along as ethnographic research tool. Ethnography 2003;4:455-85.

47. Büscher M, Urry J. Mobile Methods and the Empirical. European Journal of Social Theory 2009;12:99-116.

48. Emerson R, Fretz R, Shaw L. Writing ethnographic fieldnotes. 2nd edn. Chicago: The University of Chicago Press, 2011.

49. Clarke V, Braun V. Thematic analysis. In: Michalos AC, ed. Encyclopedia of quality of life research. New York, US: Springer, 2013.

50. Rapport F, Doel MA, Hutchings HA, et al. Eleven themes of patientcentred professionalism in community pharmacy: innovative approaches to consulting. Int J Pharm Pract 2010;18:260-8.

51. Strauss A. A social world perspective Studies in Symbolic Interaction. 1978;1:199-228.

52. Clarke A, Star S. The social worlds/arenas framework as a theorymethods package. In: Hackett E, Amsterdamska O, Lynch M, eds. Handbook of science and technology studies. Cambridge, MA: MIT Press, 2008. In. 